This item was submitted to Loughborough's Research Repository by the author.

Items in Figshare are protected by copyright, with all rights reserved, unless otherwise indicated.

\title{
Study of current distribution and oxygen diffusion in the fuel cell cathode catalyst layer through electrochemical impedance spectroscopy
}

PLEASE CITE THE PUBLISHED VERSION

http://dx.doi.org/10.1016/j.ijhydene.2012.08.141

\section{PUBLISHER}

Elsevier (@ Hydrogen Energy Publications, LLC.)

VERSION

AM (Accepted Manuscript)

\section{LICENCE}

CC BY-NC-ND 4.0

\section{REPOSITORY RECORD}

Cruz-Manzo, Samuel, Rui Chen, and Pratap Rama. 2012. "Study of Current Distribution and Oxygen Diffusion in the Fuel Cell Cathode Catalyst Layer Through Electrochemical Impedance Spectroscopy”. figshare. https://hdl.handle.net/2134/10519. 


\title{
Study of Current Distribution and Oxygen Diffusion in the Fuel Cell
}

\section{Cathode Catalyst Layer through Electrochemical Impedance}

\section{Spectroscopy}

\author{
Samuel Cruz-Manzo ${ }^{\mathrm{a}}$, Pratap Rama ${ }^{\mathrm{b}}$, Rui Chen $^{\mathrm{a}}$ \\ ${ }^{a}$ Department of Aeronautical and Automotive Engineering, Loughborough University, Leicestershire \\ LE11 3TU, UK \\ ${ }^{\mathrm{b}}$ Intelligent Energy Ltd., Loughborough, Leicestershire, LE11 3GR, UK
}

Correspondence author's current contact information:

Professor Rui Chen

Department of Aeronautical and Automotive Engineering

Loughborough University

Loughborough,

Leicestershire, LE11 3TU,

UK

Tel: +44-(0)1509-227255

Fax: +44-(0)1509-227275

e-mail: R.Chen@lboro.ac.uk 


\section{Abstract}

In this study, an analysis of the current distribution and oxygen diffusion in the Polymer Electrolyte Fuel Cell (PEFC) Cathode Catalyst Layer (CCL) has been carried out using Electrochemical Impedance Spectroscopy (EIS) measurements. Cathode EIS measurements obtained through a three-electrode configuration in the measurement system are compared with simulated EIS data from a previously-validated numerical model, which subsequently allows the diagnostics of spatio-temporal electrochemical performance of the PEFC cathode. The results show that low frequency EIS measurements commonly related to mass transport limitations are attributed to the low oxygen equilibrium concentration in the CCL-Gas Diffusion Layer (GDL) interface and the low diffusivity of oxygen through the CCL. Once the electrochemical and diffusion mechanisms of the CCL are calculated from the EIS measurements, a further analysis of the current density and oxygen concentration distributions through the CCL thickness is carried out. The results show that high ionic resistance within the $\mathrm{CCL}$ electrolyte skews the current distribution towards the membrane interface. Therefore the same average current density has to be provided by few catalyst sites near the membrane. The increase in ionic resistance results in a poor catalyst utilization through the CCL thickness. The results also show that non-steady oxygen diffusion in the CCL allows equilibrium to be established between the equilibrium oxygen concentration supplied at the GDL boundary and the surface concentration of the oxygen within the CCL. Overall, the study newly demonstrates that the developed technique can be applied to estimate the factors that influence the nature of polarization curves and to reveal the effect of kinetic, ohmic and mass transport mechanisms on current distribution through the thickness of the CCL from experimental EIS measurements.

\section{Keywords:}

Electrochemical Impedance Spectroscopy, Cathode Impedance Spectra, Cathode Impedance Model, Current distribution, Oxygen Diffusion 


\section{Introduction}

Polymer Electrolyte Fuel Cells (PEFC's) convert the chemical energy that is released during an oxygen-reduction and hydrogen-oxidation (redox) process into usable electrical energy and thermal energy. The redox process occurs electrochemically in the catalyst-based electrodes of the cell and forgoes any form of combustion; the PEFC is as such theoretically unbound by the Carnot limit. Electrochemical Impedance Spectroscopy (EIS) is an experimental technique that can be applied in-situ to separate the physical processes in the PEFC which occur at different rates through a frequency response plot. The use of equivalent electrical circuits with the experimental EIS technique is a well-established approach to characterise processes in the PEFC. Studies in the literature [1] have estimated the electrochemical and diffusion processes of the PEFC through EIS and equivalent electrical circuits. The resistances of the equivalent electrical circuit that represent the electrochemical and diffusion mechanisms of the PEFC have been compared for different operating conditions such as relative humidity $(\mathrm{RH})$, temperature, pressure, etc. and different material compositions of Gas Diffusion Layer (GDL), Micro-Porous Layer (MPL), Catalyst Layer (CL), and Polymer Electrolyte Membrane (PEM) in the PEFC.

The resulting impedance is commonly shown in a complex plane and represents the electrochemical and diffusion mechanisms of the PEFC in the frequency domain. In many EIS studies the presence of two loops in the complex plot of EIS measurements for a $\mathrm{H}_{2} /$ air PEFC have been observed. The high frequency loop has been related to the charge transfer process during the Oxygen Reduction Reaction (ORR) and the low frequency loop has been related to gas phase oxygen transport limitations in the cathode [2]. Other studies have related the low frequency loop to back diffusion of water in the membrane and diffusion of water in the catalyst layer [3]. Freire and Gonzalez [4] reported that the loop at low frequencies in EIS measurements has two contributions: the effect of liquid water formed at the cathode, which affects the transport of oxygen, and the hydration effects that limit water transport in the membrane. In some cases $[5,6]$, there is no distinct separation of the two 
loops in impedance measurements. This makes the detailed analysis more difficult and little information has been provided on the relative contribution of the charge transfer and diffusional effects under such conditions. EIS measurements have limitations and present disadvantages as the low impedance values are obscured for low frequencies and some effects are not visible due to a masking effect in the impedance plot [7]. Yuan et al. [8] attempted to separate both charge transfer and diffusional processes in the impedance spectra that account for a $\mathrm{H}_{2} /$ air PEFC stack and feature a single loop at high current densities through a Randles electrical circuit. The results showed that the Randles equivalent circuit model cannot account for the exact electrode process at high current densities because the charge transfer resistance of the ORR increased with increasing current density. Similar results have been reported by Springer et al. [2]. Mass transport effects in EIS measurements due to oxygen transport limitations are still a subject to debate as this has been principally attributed to the gaseous oxygen transport limitations in the GDL neglecting any mass transport limitations in the $\operatorname{CCL}[9,10,11]$. However, it is suspected that the CCL becomes flooded before the GDL because water is generated in the CCL and transported into the GDL, and has a lower porosity and smaller pore size, and tends to have higher flooding levels than the GDL [12].

On the other hand, there is a lack of understanding of how to interpret the frequency response of the EIS in relation to the spatio-temporal electrochemical mechanisms that occur within the PEFC. For example, by knowing exactly how the current of the ORR is distributed through the $\mathrm{CCL}$, it would be possible to develop cold starts models that include the physics of product water uptake in the membrane and filling of electrode pores with water (ice) [13]. An impedance-based approach which allows a spatio-temporal interpretation of internal fuel cell processes has not been presented or validated in the fuel cell research to date. The objective of this work is to estimate the current density and oxygen concentration distributions through the CCL thickness using EIS measurements and the mathematical model reported in the authors' previous study that accounts for the cathodic 
side of the PEFC. To validate this study, the simulated data from the model were compared with Cathode EIS measurements in Bode format (modulus and phase) and impedance format (real, imaginary) obtained in a three-electrode configuration in the measurement system.

\section{Model Description}

In the authors' previous study [14,15], a model was developed based on fundamental electrochemical and diffusion theory to simulate the impedance spectrum of the CCL operated for any zone of the polarization curve, and also the current and oxygen distributions through the $\mathrm{CCL}$ in the spatio-temporal domain. The theoretical treatment considered the following main assumptions;

a. only effects in the CCL are considered,

b. all processes proceed under isobaric and isothermal conditions,

c. spatial gradients are only considered through the thickness of the $\mathrm{CCL}$,

d. the resistance to ion transfer in the electrolyte of $C C L$ is much greater than the resistance to the electron transfer in the carbon of the catalyst layer. Therefore electronic ohmic loss in CCL can be regarded as being negligible $[16,17,18]$

e. the rate of oxygen distribution is considered to be a non-steady process.

In the model, fundamental electrode theory is applied to define the equation for low current distribution in the CCL. Fick's second law in the frequency domain is solved and applied to define oxygen distribution through $\mathrm{CCL}$. The rate of oxygen distribution and ionic conductivity are related to a low current distribution equation for the CCL. The simulation 
results in the frequency domain are compared against measurements from impedance spectra, which are then converted into the time domain using the inverse Laplace transform method. In the time domain the model simulates the current distribution through the $\mathrm{CCL}$ thickness, and in general can be applied for any region of the polarization curve (low, mid, high current). The use of equivalent electrical circuits with the experimental EIS technique is a well-established methodology to characterize processes in the PEFC [19]. The electrical circuit that models the cathode impedance spectrum generated from EIS analysis and obtained through a reference electrode contains an inductor element that accounts for the inductance of the cables used in the test equipment connected in series with a resistance that accounts for the PEM, GDL and bipolar plate. They are connected in series with a circuit that accounts for the CCL, in which for this specific case is the CCL impedance equation reported in the previous work [14],

$Z_{C}=L(i \omega)+R_{e}+\frac{\left(R_{C}+Z_{W}\right) \gamma \operatorname{coth}(\gamma x)}{1+Y(i \omega)^{P}\left(R_{C}+Z_{W}\right)}$

where $\gamma=\sqrt{R_{p}\left[\frac{1}{R_{C}+Z_{W}}+Y(i \omega)^{p}\right]}$,

$R_{P} \quad$ is the resistance to the flow of ions in the electrolytic phase of the CCL,

$R_{c} \quad$ represents the charge transfer resistance presented in the ORR and is defined as $R_{C}=b / j_{0} \exp \left(\eta_{S} / b\right)$, where $b$ is the Tafel slope, $\eta_{s}$ represents a value of voltage in activation overpotential, and $j_{0}$ is the exchange current,

$Z_{W} \quad$ is defined as the Warburg impedance and describes diffusion across a finite dimension in the frequency domain [20] $Z_{W}=R_{W} \tanh \left(i \omega T_{W}\right)^{0.5} /\left(i \omega T_{W}\right)^{0.5}$, with $R_{W}=R T \delta /\left(z^{2} F^{2} c_{o}^{*} D\right)$ defined as resistance for the diffusion process and $T_{W}=\delta^{2} / D$ defined as the time constant to diffuse oxygen through the CCL, 
$Y(i \omega)^{P} \quad Y$ represents a parameter related to constant phase element (CPE), superscript $P$ represents a parameter to correct the inhomogeneity in the distribution of charge,

$\omega$ is the angular frequency,

$i \quad$ is the imaginary component in impedance,

$L \quad$ represents the inductance in the electrical cables of the measurement system,

$R_{e} \quad$ represents the total ohmic resistance to flow electrons and ions in the bipolar plate, GDL, PEM.

\section{Experimental}

EIS is commonly applied as a single channel through a two-electrode configuration. A twoelectrode configuration makes it difficult to identify, to separate and to reveal the process related to each electrode (anode, cathode) in the impedance spectrum of a cell. A threeelectrode configuration in EIS allows the measurement of half-cell impedance (anode or cathode) to be carried out. Some studies [21,22] have applied the three-electrode configuration in EIS measurements. Under such conditions, the signals are measured between the working electrode (WE) and the reference electrode (RE), and the current induced is collected by the counter electrode (CE).

A $25 \mathrm{~cm}^{2}$ commercially-available fuel cell and test rig acquired from Baltic Fuel Cells were used for the experimental tests. The MEA was a membrane DuPont Nafion-115 with a platinum loading of $0.4 \mathrm{mg} / \mathrm{cm}^{2}$ on both electrodes. The operational temperature was $50^{\circ} \mathrm{C}$ and the gauge back gas pressure was held to 0.9 bar for both the anode and cathode. Flow 
rates were held constant during all the experiments, hydrogen to the anode was supplied at a stoichiometry of 2.5 and air to the cathode supplied at a stoichiometry of 3 . The PEFC was operated with $100 \%$ hydrogen relative humidity $(\mathrm{RH})$ in the anode, the air supplied in the cathode was dry. Qiangu Yan [23] reported that the best performance in a PEFC occurs at low air relative humidity and high hydrogen relative humidity. EIS measurements were carried out through a multichannel Frequency Response Analyser (FRA) (Z\#106 WonATech Co). The multichannel FRA consists of five channels and simultaneously measures five impedance spectra through a one DC current value induced. EIS measurements were carried out at three different current densities $0.12,0.2$ and $0.32 \mathrm{~A} / \mathrm{cm}^{2}$. The multichannel system is connected with a RBL488 Dynaload. The multichannel FRA superimposes a small AC sinusoidal perturbation onto the bias current induced by the RBL488 Dynaload unit and measures the AC voltage signals resulting from the PEFC. The impedance measurements were carried out in a galvanostatic mode with a 5\% AC amplitude of the DC current $[8,24,25]$ to obtain a linear response from the system and at frequencies from $10 \mathrm{KHz}$ to $0.3 \mathrm{~Hz}$. The high frequency in the EIS measurements was limited due to the bandwidth of the electronic load. Scattered data in the EIS measurements were manifested at high current densities and at frequencies lower than $0.3 \mathrm{~Hz}$. The air gas reactant supplied in the cathode was dry and the water produced by the ORR ensured an optimal hydrated state in the CCL and PEM. However at low frequencies $<0.3 \mathrm{~Hz}$ during the EIS measurements the high water concentration in the cathode lead to instability in the PEFC. In the previous authors' study [26], it was demonstrated that the inductance of the electrical cables of the measurement system deforms the high frequency region of the PEFC spectrum and as such leads to an incorrect interpretation of the electrochemical mechanisms at high frequencies. In this study, special electrical cables (Low inductive cable with fusion Lug Technology, TDI POWER) connected between the PEFC and the RBL488 Load were used to reduce inductive effects on the EIS measurements. To separate the impedance spectrum of the cathode from the impedance spectrum of the cell, a reference electrode was inserted such that it was in direct contact with the membrane of the cathode side. Two channels from the Z\#106 FRA were 
used to simultaneously measure the impedance of the PEFC and the impedance solely accounting for the cathode electrode.

The resulting impedance is commonly shown in a complex plane and represents the electrochemical and diffusion mechanisms of the PEFC in the frequency domain. It has been proposed that the PEFC impedance spectrum largely represents the electrochemical processes in the cathode, and because the electrochemical mechanisms in the anode are fast and facile are not expected to have a contribution and therefore it can be regarded as negligible $[20,27]$. This theory will not always be valid as the transport of water from the anode to the cathode by electro-osmotic drag can play a role in dehydrating the membrane and inducing performance degradation [28].

The diameter of the spectra accounting for PEFC and Cathode increases with increasing current density, as shown in Fig.1a. When the kinetics of the ORR dominates the cell performance such as in the low current density range of the polarization curve, the impedance spectrum mainly represents the charge transfer effect during the ORR and its diameter decreases with increasing current density [7]. In Fig. 1a, the increase in diameter of the spectra with increasing current density can be attributed to an increase in oxygen transport limitations. One of the disadvantages of the EIS technique is that multiple energy controlled process during the electrochemical reaction can be masked in the impedance plot. The impedance results of Fig. 1a reflect the overlapping of two semicircles. One is related to the charge transfer of the ORR effect which decreases with increasing current density and the other is related to mass transport effect which increases with increasing the current density. The semi-circular part of the cathode spectra presents roughly the same features as the PEFC spectra and a shift over the real part $Z$ ' $\Omega . c m^{2}$. This effect is expected as the Cathode is hydrated by the water produced by the ORR and due to electro-osmotic drag from the anode. The difference between the PEFC and Cathode spectra can be attributed to the electro-osmotic effect from the anode. The Cathode and PEFC spectra at current densities of 0.2 and $0.32 \mathrm{~A} / \mathrm{cm}^{2}$ intercept the real component $Z^{\prime} \Omega . \mathrm{cm}^{2}$ at almost the same 
position, as shown in Fig $1 \mathrm{~b}$; this suggests that the PEM and CCL are fully hydrated at 0.2 $\mathrm{A} / \mathrm{cm}^{2}$ so an increase in current density leads to an increase in oxygen transport limitations.

\section{Results and Discussion}

\subsection{Cathode impedance validation}

In these experimental results the use of a reference electrode in the measurement system ensures that the data accounting for the processes in the Cathode are captured for analysis and interpretation. The overall modelling and experimental framework developed for the current study is illustrated in Fig. 2. The modelling work involves the simulation of the Cathode impedance through Eq. 1 and using a graphic user interface (GUI) developed in Matlab®. In Eq. 1 of the model there are some parameters that can be estimated through a graphical interpretation of the complex plot. This allowed the reduction of the number of parameters to be fitted in the measured EIS spectra. The high frequency limit of the real part $Z^{\prime}$ of the impedance spectrum is given by a resistance $R_{e}$ accounting for ohmic loses in the PEM, GDL, and bipolar plate [29]. In the authors' previous study [26], it was demonstrated that if the inductance of the measurements cables is reduced, the ionic resistance $R_{P}$ in the $\mathrm{CCL}$ electrolyte can be represented in the cathode impedance spectrum by a $45^{\circ}$ straight line at the high frequency end of the spectrum and its value over the real part of the impedance spectrum is calculated as $R_{p} / 3$. Ciureanu, et al. [27] reported that the double layer capacitance is calculated as: $C=Y /\left(R_{e}^{-1}+R_{c}^{-1}\right)^{1-P}$, and Hsu [30] defined the capacitance as: $C=Y\left(2 \pi f_{C}\right)^{P-1}$, nothing that $f_{C}$ is the characteristic frequency at which the negative imaginary part of the impedance reaches its maximum value. The CCL oxygen diffusion time constant $T_{W}$ was fixed at 3ms as reported by Springer et al. [2] to avoid inclusion of another fitting variable. The charge transfer resistance $R c$ and mass transport resistance $R_{W}$ whose contributions are at medium and low frequencies respectively were the only parameters used in the fitting process. This provides assurance that the parameters in Eq. 1 correctly 
relate to the physics and chemistry that are occurring. The experimental work provides the measured frequency response of the cathode for validation purposes. The least squares fitting method was used in order to find the best-fit between the model and the measured data. A good quality fit is obtained when the sum of the deviations squared (least square error) between the simulated and measure impedance data has a minimum value, for instance $<0.1$.

In Fig. 3, the results show a good agreement between the measured and simulated data in the complex-plot with an exception at the highest frequencies $(>7 \mathrm{KHz})$ where the measured data exhibit a scatter due to high frequency noise presented during the EIS test and due to external inductance of the measurement system. In the authors' previous study [26], it was demonstrated that the property of causality in the Kramers-Kronig mathematical relations for Electrochemical Impedance Spectroscopy (EIS) measurements is violated by the external inductance of the measurement cables and external noise during EIS. Thereby the measured data at the highest frequencies (scattered data) in Fig. 3 do not represent the physics and chemistry of the Cathode. The model of Eq. 1 is based on differential equations describing the detail of the physical processes in the cathode $[14,15]$, therefore the comparison between measured and simulated data provides a good validation of the measured data, rather than evaluating the data to an analysis of Kramers-Kronig consistency.

A further validation of the mathematical model against the modulus and phase angle of the frequency response of the measured data was carried out. Sensitivity to discrepancies between model and experimental data can be assessed by comparing the experimental data with the simulated data in a Bode format (modulus and phase). Orazem [31] reported in his study that the Bode modulus and real part component of the impedance plots are relatively insensitive to the quality of the fit of a model to impedance data. The imaginary component of the impedance and Bode phase angle plots are modestly sensitive to fit quality. 
Figs. 4a,b show the comparison between measured and simulated data in Bode format. Fig. 4a shows that the model is able to reproduce accurately the Bode modulus for all the frequencies. Fig. 4b shows a discrepancy in the phase angle between measured and simulated data at the highest frequencies $(>7 \mathrm{KHz})$. The model cannot reproduce the measured phase angle in the frequency range of $7 \mathrm{KHz}$ to $10 \mathrm{KHz}$ because the measured data over this frequency range do not represent the physics and chemistry of the electrochemical system, as discussed previously. Fig. 4a shows that the modulus at 0.2 and $0.32 \mathrm{~A} / \mathrm{cm}^{2}$ show a similar tendency from $10 \mathrm{KHz}$ up to $\sim 6 \mathrm{~Hz}$. The modulus from $10 \mathrm{KHz}$ to $6 \mathrm{~Hz}$ can be related to the hydrated state of the CCL and PEM which indicates that $\mathrm{CCL}$ and PEM at 0.2 and $0.32 \mathrm{~A} / \mathrm{cm}^{2}$ are fully hydrated due to the water produced by the ORR. The asymptotic value at high Frequency $(10 \mathrm{KHz})$ provides a qualitative value of the ionic resistance in the PEM and CCL which decreases at current densities of 0.2 and $0.32 \mathrm{~A} / \mathrm{cm}^{2}$. The asymptotic value of the modulus at low frequency of $0.3 \mathrm{~Hz}$ provides a qualitative value of the mass transport resistance which increases with increasing current density. In Fig. 4b the phase angle for the three current densities presents the same tendency from $10 \mathrm{KHz} u p$ to $\sim 120 \mathrm{~Hz}$. The phase angle tends toward zero with decreasing the frequency $<0.3 \mathrm{~Hz}$ for the three cases, indicating that the current and potential become into phase as frequency approaches zero. An out of phase shift for the three cases is clearly shown in Fig. 4b. The single peak in the phase angle plot suggests that a single time constant describes the electrochemical process; multiple peaks would provide a clear indication that more than one time constant is required to describe the electrochemical process during the ORR [31]. In Fig. $4 b$, the frequency, where the phase angle reaches its minimum value, decreases with increasing current density. This reduction in frequency suggests that the process required to control the ORR becomes slower with increasing current density. Overall, the simulated data demonstrates that the mathematical model shown in Eq. 1 can be reliably applied in order to reproduce the measured data obtained at the three different current densities. 


\subsection{Estimation of electrochemical and diffusion mechanisms}

The mathematical model represented by Eq. 1 allows the estimation of the ionic resistance $R_{P}$ of the CCL electrolyte, charge transfer resistance $R_{C}$ of the ORR and mass transport resistance $R_{W}$ due to oxygen limitations in the cathode from EIS measurements. The ionic resistance in the $\mathrm{CCL}$ electrolyte decreases with increasing current density, as shown in Tab. 1. This effect is attributed to the water produced during the ORR and the water transported from anode to cathode by electro-osmotic drag. The charge transfer resistance, whose dependence on electrode potential is given by the Tafel equation and reflects the increase in the driving force for the interfacial oxygen reduction process, decreases with increasing current density. The mass transport resistance, due to gaseous oxygen transport limitations and as a function of the gaseous oxygen equilibrium concentration $c_{O}^{*}$ in the $\mathrm{CCL}$ GDL interface, increases with increasing current density, as shown in Tab.1. Mass transport resistance is a minimum at $0.12 \mathrm{~A} / \mathrm{cm}^{2}$ due to the low water concentration produced during the ORR, which improves the ability of oxygen to permeate to the active sites of $\mathrm{CCL}$; nevertheless low water concentration increases the ionic resistance in the CCL. At current density of $0.2 \mathrm{~A} / \mathrm{cm}^{2}$, both charge transfer and mass transport resistances have roughly the same magnitude therefore under such a current density the balance between the electrochemical and diffusion processes is achieved. At current density of $0.32 \mathrm{~A} / \mathrm{cm}^{2}$, the mass transport effect dominates the performance of the PEFC.

Mass transport limitations have been principally attributed to the gaseous oxygen transport limitations in the GDL neglecting any mass transport limitations in the $\operatorname{CCL}[9,10,11]$. However, it is suspected that the CCL becomes flooded before the GDL. Mass transport limitations represented in the complex plot of EIS measurements have been a subject to debate.

Oxygen Permeability Through CCL - The catalyst layer is commonly formed by a composite structure of a matrix of carbon grains providing the electron conductivity, $\mathrm{Pt}$ 
supported on carbon as the catalyst, Teflon (PTFE) as a binder that stabilizes the carbon matrix and as a hydrophobizing agent, and electrolyte network of perfluorosulfonate ionomer (PFSI) soaked with water. The matrix of carbon grains forms agglomerate structures. Primary pores are those that exist inside the agglomerates between the carbon grains. Secondary pores constitute the void spaces between agglomerates. There are mainly three principal modes of transport of $\mathrm{O}_{2}$ in the CCL: gas-phase diffusion in the CCL pore (primary, secondary), diffusion in the liquid-water film surrounding catalytic agglomerate, and diffusion in the ionomer of the agglomerate. The analysis of this study assumes that the catalyst agglomerated is covered by a film of liquid water and Nafion is homogeneously spread through the thickness of the CCL. Springer et al. [32] defined and estimate the permeability of oxygen in the CCL through EIS measurements. The permeability of oxygen in the CCL $D c_{o}^{*}$ was defined by the product of the effective diffusion coefficient of oxygen and the equilibrium oxygen concentration in the catalyst layer. Their EIS results showed oxygen permeabilities of the order of magnitude $10^{-10}$ for a catalyst layer with $5 \mu \mathrm{m}$ thickness of a $5 \mathrm{~cm}^{2} \mathrm{H}_{2}$ /air PEFC. This author concluded that a low value for the oxygen permeability is required to generate the break in the cell polarization curve. The thickness of the catalyst layer used in the EIS measurements in this study is $12 \mu \mathrm{m}$ and the oxygen permeability in the $\mathrm{CCL}$ calculated from EIS measurements and through Eq. 1 are $D c_{O}^{*}=1.92 \times 10^{-10}, 4.86 \times 10^{-11}, 2.23 \times 10^{-11} \mathrm{~mol} / \mathrm{cm} . \mathrm{sec}$ for $0.12,0.2$ and $0.32 \mathrm{~A} / \mathrm{cm}^{2}$ respectively. The decrease of the oxygen permeability reflects a limitation in transport routes for oxygen in the CCL.

Time Constant of Oxygen Diffusion Through CCL - The single peak in the phase angle of EIS measurements shown in Fig. $4 \mathrm{~b}$ reflects the fact that a single energy processes controls the ORR. The single loop of the EIS measurements shown in Fig. 3a reflects the same assumption. However this may not always be the case as the capacitance related to the charge transfer process of the ORR and the oxygen diffusion effect are normally represented together in EIS measurements. One of the disadvantages of the EIS technique is that the 
frequency dependency is obscured because multiple energy controlled process of an electrochemical system can be masked in the frequency plot. Based on the results from Fig. 3a, the mass transport effect was overlapped with the charge transfer effect in the EIS measurements. Similar results were reported by Yuan et al. [8] when a $280 \mathrm{~cm}^{2}$ PEFC stack was operated with a hydrogen stoichiometry of 2 , and an air flow stoichiometry of 2.5 . In the study carried out by Yuan et al, the separation between the charge transfer process and mass transport effect in EIS measurements was apparent when the PEFC stack was operated with a hydrogen stoichiometry of 2 and a fixed air flow rate of 20 standard $L \mathrm{~min}^{-1}$ (low air flow stoichiometry). Ciureanu et al. [27] carried out EIS measurements in a $25 \mathrm{~cm}^{2}$ PEFC with flow rates at stoichiometry of 1.2 hydrogen and 2 air. The results show the additional loop at low frequencies that accounts for oxygen transport limitations in the cathode. Other studies have reported the presence of two loops in the EIS measurements where that at low frequencies reflects the oxygen transport limitations in the gas phase because of the shortage of the air supply operated at a fixed air flow rate. Within the fuel cell there is a competition mainly between back-transport of water from cathode to anode and electro-osmotic drag of water by ions from anode to cathode. If no other source or sink of water is considered, the ideal operating point needs to be where both fluxes are equal. If the $\mathrm{H}_{2}$ /air PEFC is operated with a low air flow rate the semicircle that represents the mass transport limitations at low frequencies is visualized in the EIS measurements. In the authors' previous study [14], it was demonstrated that the mathematical model represented in Eq. 1 is able to reproduce EIS measurements resulting in the low frequency loop attributed to gaseous oxygen transport limitations in the cathode.

The time constant to diffuse oxygen through the CCL expressed in Eq. 1, is defined by $T_{W}=\delta^{2} / D$ where $\delta$ represents the characteristic length scale of the diffusive process in the $\mathrm{CCL}$, in which for this specific case is considered to be the thickness of the CCL and $D$ represents the effective diffusion coefficient for oxygen transport in the CCL. Springer et al. [2] reported time constants to diffuse oxygen through the CCL not larger than $3 m s$ using a 
mathematical model fitted with EIS measurements for a $5 \mathrm{~cm}^{2} \mathrm{H}_{2} /$ air PEFC. The time constant in their study was fixed at $3 m s$ for all EIS measurements to avoid inclusion of another fitting variable. The time constant for the validation in this study was fixed a constant value of $3 m s$, as reported by Springer, for the three EIS measurements, as shown in Tab.1. The effective diffusion and equilibrium oxygen concentration estimated from Eq. 1 were $D=4.8 \times 10^{-4} \mathrm{~cm}^{2} / \mathrm{sec}$ and $c_{O}^{*}=4.008 \times 10^{-7}, 1.013 \times 10^{-7}, 4.651 \times 10^{-8} \mathrm{~mol} / \mathrm{cm}^{3}$ for $0.12,0.2$ and $0.32 \mathrm{~A} / \mathrm{cm}^{2}$ respectively. The time constant $T_{W}=0.003 \mathrm{sec}$ from Tab. 1 was increased in two orders of magnitude in Eq. 1 by decreasing in two orders of magnitude the effective diffusion coefficient to simulate its effect on the EIS measurements. The remaining parameters from Eq. 1 were kept constant.

The semicircles that represent the charge transfer of the ORR and mass transport effects are overlapped in the experimental cathode spectra, as shown in Fig. 5. The results in Fig. 5 show that if the time constant $T_{W}=3 m s$ from Tab. 1 is increased by two orders of magnitude in Eq.1, it is possible to separate at $3.33 \mathrm{~Hz}$ the semicircles that represent charge transfer effect and oxygen transport limitations in the cathode spectra and whose diameters reflect roughly the same value as the $R c$ and $R_{W}$ from Tab. 1. Springer et al. [2] concluded that it is not possible to visualize the charge transfer effect and mass transport effect in PEFC EIS measurements with time constants of $10^{-4}, 10^{-3}, 10^{-2}$ orders of magnitude, and with either air or oxygen as a gas reactant.

Malevich et al. [33] reported in their study the effective diffusivity of oxygen in gas phase and ionomer phase of the CCL to be $2.68 \times 10^{-4} \mathrm{~cm}^{2} / \mathrm{s}$ and $2.83 \times 10^{-6} \mathrm{~cm}^{2} / \mathrm{s}$, respectively. These values have the same order of magnitude as the CCL oxygen diffusion estimated for time constants 0.003 and $0.3 \mathrm{sec}$ in this study. It can be concluded from the EIS Cathode measurements of Fig. 3 with time constants of $0.003 \mathrm{sec}$ and CCL oxygen diffusion constant of $4.8 \times 10^{-4} \mathrm{~cm}^{2} / \mathrm{s}$ that the main contribution to oxygen transport is through the porous media of the CCL. The simulated Cathode spectra in Fig. 5 with a time constant of $0.3 \mathrm{sec}$ and a 
diffusion of $4.8 \times 10^{-6} \mathrm{~cm}^{2} / \mathrm{s}$ suggest that the main contribution to oxygen transport is through the ionomer surrounding the agglomerate in the CCL. Because this is a macroscopic modelling approach, it is not possible to separate transport mechanism contributions due to small pores between agglomerates in which the Knudsen flow would be expected to be the prevailing mechanism and due to macropores in the void space between the agglomerates where a molecular diffusion would be the dominant transport mechanism. Water saturation in the CCL is expected when the PEFC is fed at low flow rates. Low flow rates result in the decrease of the effective porosity in the CCL due to the accumulation of water. Under such condition an increase in the CCL diffusion time constant is expected. Malevich et al. [33] also reported diffusive time constants with a magnitude order of $10^{-1} \mathrm{sec}$ in EIS measurements of a PEFC stack with MPLs adjacent to the GDLs. The EIS measurements clearly showed the mass transport loop at low frequencies but with a diameter that is relatively smaller than the charge transfer loop. The MPL can reduce water saturation in the GDL and promote the water back to the CCL. Under this condition a saturation of liquid water in the $\mathrm{CCL}$ is expected. The mass transport resistance $R_{W}$, shown in Tab. 1 , is a function of the gaseous oxygen equilibrium concentration $c_{O}^{*}$ in the CCL-GDL interface; a MPL enhances the gaseous oxygen transport through the GDL and therefore increases the equilibrium oxygen concentration at the CCL-GDL interface. Hence EIS measurements carried out in a PEFC with MPL's present lower mass transport resistance than for cells without MPL's. However under this condition, an increase in the time constant for the diffusion process of oxygen in the CCL is expected because the diffusivity of gaseous oxygen transport in the CCL is reduced due to the higher water concentration.

In cells without MPL's as the case of this study, an increase in amount of water due to the ORR is expected in the GDL with increasing current density. It can be concluded that the increase of mass transport resistance as shown in Tab. 1 and represented as the diameter of the EIS loop at low frequencies is related to the low equilibrium oxygen concentration in the CCL-GDL interface as water is blocking the path for oxygen to permeate through the 
GDL, and the time constant for the diffusion process in EIS measurements is related to the oxygen transport through the composite structure in the CCL.

\subsection{Oxygen and current distributions through CCL}

Oxygen distribution through $\mathrm{CCL}$ - The effect of oxygen transport on the CCL at the frequency domain is commonly represented in EIS measurements at low frequencies. Therefore the oxygen transport through the CCL occurs in a non-stationary manner in the time domain. The effective diffusion coefficient of oxygen in the CCL calculated from the time diffusion constant was the same for the three current densities $D=4.8 \times 10^{-4} \mathrm{~cm}^{2} / \mathrm{sec}$ and the equilibrium oxygen concentration at the CCL-GDL interface was $c_{0}^{*}=4.008 \times 10^{-7}, 1.013 \times 10^{-7}, 4.651 \times 10^{-8} \mathrm{~mol} / \mathrm{cm}^{3}$, for $0.12 \mathrm{~A} / \mathrm{cm}^{2}, 0.2 \mathrm{~A} / \mathrm{cm}^{2}, 0.32 \mathrm{~A} / \mathrm{cm}^{2}$ respectively. The oxygen concentration distribution through the $\mathrm{CCL}$ in the time domain was calculated using a model presented elsewhere [14]. The analysis is carried out at a CCL thickness fraction of $0.25,0.45$ and 0.75 , where $x=0$ is the thickness fraction at the GDL-CCL interface and $x=1$ is the thickness fraction at CCL-PEM interface.

The oxygen concentration at $t=0$, represents the equilibrium oxygen concentration at the CCL-GDL interface which at a current density of $0.12 \mathrm{~A} / \mathrm{cm}^{2}$ has a high value due to the low water concentration and the facility for oxygen to diffuse through the GDL, as shown in Fig. 6a. Fig. $6 a$ also shows that at $0.12 \mathrm{~A} / \mathrm{cm}^{2}$ where mass transport limitation is minimum there is a minimum effect on the concentration distribution through the CCL thickness. Figs. $6 b, c$ shows an initial depletion of oxygen concentration through the CCL thickness and more severe depletion at the $x=0.75$ thickness fraction and current density of $0.32 \mathrm{~A} / \mathrm{cm}^{2}$. This occurs due to the ORR as current is established in the CCL and the process takes place in the order of $1 \mathrm{~ms}$. When the oxygen concentration presents a value of zero in the CCL, a break in the cell polarization curve is generated. The results show that the diffusion of oxygen through the CCL occurs in a non-stationay manner and recovers to the equilibrium oxygen concentration value throughout the CCL at steady-state, which occurs in $0.003 \mathrm{sec}$., 
as shown in Figs. 6b,c. It is noteworthy that this value corresponds to the same value of the CCL diffusive time constant from the EIS measurements, Tab.1. This recovery of oxygen concentration to the equilibrium oxygen concentration at the GDL-CCL interface will depend on CCL structure (porosity, tortuosity), the water removal characteristics of the adjacent porous layers (GDL, MPL), cell operating conditions, and the contact pressure in the MEA assembly.

Current distribution through CCL - In order to improve the performance of the electrode as well as to determine the membrane uptake of product water, it is important to know and to optimise the current distributions within the CCL. In the authors' previous study [14], the current distribution through the CCL based on EIS measurements and fundamental electrochemistry and diffusion theory was defined.

$\frac{d^{2} \bar{j}}{d x^{2}}=R_{P}\left[\frac{\bar{j}}{R_{C}+R_{M}}+C \frac{d \bar{j}}{d t}\right]$

where $R_{M}=\left(8 / \pi^{2}\right) \exp \left(-\pi^{2} D t / 4 \delta^{2}\right) R_{W}$, and $R_{W}=R T \delta / z^{2} F^{2} C_{o}^{*} D$. The parameters from Tab.1 were applied to simulate the current distribution through the CCL thickness. The current distribution through the CCL thickness occurs in a transitory manner due to the nonstationary oxygen diffusion process in the $\mathrm{CCL}$, as shown in Fig. 6. It has been previously [14] demonstrated that the current distribution shows an initial transitory response due to the ionic resistance and the capacitance for all CCL thicknesses and this initial response is also related to the interfacial area between the electrode and electrolyte network. Thereafter the transitory current distribution in the $\mathrm{CCL}$ achieves a maximum current as a function of the ionic resistance, charge transfer resistance and mass transport resistance under potentiostatic conditions. The maximum current achieved is a function of the maximum depletion of oxygen concentration. The maximum current also reflects the rate at which oxygen can be brought to the electrode surface for a given set of mass transfer conditions. Then, the increase in current density is proportional to the decrease in oxygen 
concentration in the CCL. Subsequently there is a decrease in the current distribution due to the nonstationary oxygen diffusion process in the CCL [14]. The current distribution achieves steady state by $0.003 \mathrm{sec}$, and the magnitude of the steady-state current depends only on the ionic resistance and charge transfer resistance. To achieve the current distribution in which mass transport resistance is neglected it will depend on operating conditions (flooding, drying), contact pressure in the MEA assembly, CCL structure and the operational gas pressure.

In this study, the maximum current distribution in the $C C L$ which reflects the maximum depletion of oxygen through the $\mathrm{CCL}$ and is a function of the ionic resistance, charge transfer resistance and mass transport resistance is considered. This point of the transitory current distribution was chosen as this reflects the polarization curve with the three overpotentials (activation, ohmic, concentration). The ideal operating point of the current distribution would be where mass transport effect is negligible, however this effect depends on the oxygen equilibrium concentration and diffusion constant, parameters which can be influenced by operation and preparation of the fuel cell. The equation for the current distribution in Eq. 3 is defined by the ratio of the ionic resistance to the sum of the charge transfer resistance and the mass transport resistance in the time domain. Neyerling [34] defined a similar relationship in the absence of the mass transport resistance, where the higher the dimensional ratio is, the more the current distribution will be skewed toward the membrane interface, while low values of this ratio predict a homogeneous current distribution. The ionic resistance, charge transfer resistance and mass transport resistance in the CCL can change for different operating conditions. In this study, if the ratio between ionic resistance and the sum of both the charge transfer resistance and mass transport resistance is increased, the ions may react only closer to the membrane. However, in the case when the ratio is reduced, the ions will react through the entire CCL thickness leading to a uniform current distribution. 
The current distribution (area below the curve) through the CCL increases from the $x=0$ thickness fraction (GDL-CCL interface) to the $x=1$ thickness fraction (CCL-PEM interface), as shown in Fig. 7. This reflects the transfer of ions from the PEM and the anode catalyst layer, which results in a high value of current at the interface between the $C C L$ and the PEM and a minimum current at the interface between the CCL and GDL as ions are consumed due to the ORR through the thickness of the CCL. A high ionic resistance within the CCL electrolyte tends to skew the current distribution towards the membrane interface, $(0.12$ $\left.\mathrm{A} / \mathrm{cm}^{2}\right)$. Therefore the same average current has to be provided by fewer catalyst sites near the membrane. The increase in ionic resistance results in greater activation loses. Fig. 7 shows that the current density of $0.12 \mathrm{~A} / \mathrm{cm}^{2}$ is generated from $x=0.449$ to $x=1$, for $0.2 \mathrm{~A} / \mathrm{cm}^{2}$ is generated from $x=0.3265$ to $x=1$, and for $0.32 \mathrm{~A} / \mathrm{cm}^{2}$ from $x=0.2245$ to $x=1 \mathrm{CCL}$ thickness fraction. Therefore at high current densities there is an increase in the utilization of the catalytic particles through the CCL thickness because the ORR current distribution follows Tafel kinetics. This is also supported by the decrease in charge transfer resistance from Tab.1 when increasing current density. The ORR current distribution is further influenced by the mass transport resistance of oxygen reactant diffusion into the CCL. Taijiri et al. [35] studied the phenomenon of gas phase diffusion of oxygen at subfreezing conditions, and they concluded that the current distribution was dominated by the gas transport effect without estimating voltage losses in the CCL. Thompson et al. [13] studied the current distribution through the CCL without considering mass transport resistance of oxygen at subzero conditions of $-20^{\circ} \mathrm{C}$. This author concluded that the mass transport effect would skew the current distribution toward the diffusion medium interface (CCL-GDL). Overall, in Fig. 7 it is noticeable that the increase of mass transport resistance from Tab. 1 tends to skew the ORR current distribution towards the GDL interface. These valuable impedance-based results of ORR current distribution will help to develop cold-start models that include the physics of membrane uptake of product water and filling of electrode pores with water (ice) [12]. 
Discussion - The oxygen diffusion coefficient in the CCL can be adversely affected by CCL structure, composition and operating conditions. It is clearly known that by reducing the void space between agglomerates due to the accumulation of water the ability of oxygen to permeate into the active sites of the CCL will be affected. There are two more modes of transport of oxygen in the CCL which can have an effect on the ORR: dissolved oxygen diffusion in the liquid water surrounding the agglomerate, and dissolved oxygen in the ionomer phase. To date, the effect of the oxygen diffusion on every single phase of the CCL material through impedance measurements has not been studied, and the literature mainly considers the gas phase diffusion as the crucial factor of the mass transport limitations.

One of the challenges in the impedance technique is to separate the mass transport limitations between the CCL and the GDL. Equivalent electrical circuits with Warburg electrical component have been broadly used in PEFC impedance measurements to estimate the mass transport limitations. However it has not been clear how to relate the effect of oxygen transport limitation in the CCL and GDL. As for a higher water content in the CCL the mass transport limitations in the GDL would be reduced and vice-versa. Mass transport in PEFC impedance measurements have been commonly attributed to oxygen transport limitations in the GDL neglecting any mass transport effect in the CCL. This study has demonstrated that mass transport limitations represented in EIS measurements are related to the equilibrium oxygen concentration at the GDL-CCL interface and the diffusivity of oxygen through the composite structure in the CCL.

This study has demonstrated that it is possible to estimate the current density and oxygen concentration distributions in the $\mathrm{CCL}$ by coupling the fundamental electrochemistry and diffusion theory of the CCL to the experimental impedance technique. The results obtained can give an insight about the physics of product water uptake in the membrane and formation of ice in the CCL during subfreezing operation. In terms of design and optimisation of the CCL, it is necessary to carry out further analysis on the effective diffusion of oxygen through the CCL as a multi-phase system. This could be achieved through the theoretical 
work developed to study the local inhomogeneities in the effective oxygen diffusion through a segmented CCL and with the use of a multichannel impedance system. This is the aim of a future work.

\section{Conclusions}

EIS measurements that account for the cathodic side of the PEFC were compared with simulated data obtained through the Cathode Impedance model developed in the authors' previous study. A validation in the complex-plot and bode format was carried out to account for the exact electrode process in the Cathode EIS measurements. The results showed that the low frequency loop in PEFC impedance measurements that commonly attributed to mass transport limitations is attributed to the oxygen equilibrium concentration in the CCLGDL interface and the diffusivity of oxygen through the CCL.

A further analysis on the current density and oxygen concentration distributions through the $\mathrm{CCL}$ thickness was evaluated. The results showed that the diffusion of oxygen through the CCL occurs in a non-stationary manner and recovers to the equilibrium oxygen concentration value throughout the $\mathrm{CCL}$. This recovery of oxygen concentration to the equilibrium oxygen concentration at the GDL-CCL interface will depend on CCL structure, the water removal characteristics of the adjacent porous layers, cell operating conditions, and the contact pressure in the MEA assembly.

The results also showed that at high current densities (low ionic resistance, high mass transport resistance) the ORR current distribution which follows Tafel kinetics is skewed towards the CCL-GDL interface improving the catalyst utilization through the CCL thickness. This newly established EIS knowledge will enable an assessment of the state of health of operational fuel cells and enhance cell development in terms of physical changes within fuel cell materials and the critical operational factors through non-intrusive diagnostics. 
This study has demonstrated that EIS is a powerful tool for in-situ diagnosis of a PEFC. It is possible to generate a deeper understanding of the internal phenomenological process by coupling the experimental EIS technique to the fundamental theory.

\section{Acknowledgments}

The authors thank the Mexican National Council for Science and Technology (CONACYT) for the sponsorship of the Ph.D research study of S. Cruz-Manzo (Grant no. 183195). 


\begin{tabular}{lcccc}
\hline $\begin{array}{l}\text { Current } \\
\text { Density }\end{array}$ & $\mathbf{R}_{\mathrm{P}} \Omega . \mathrm{cm}^{2}$ & $\mathbf{R}_{\mathrm{C}} \mathbf{\Omega} . \mathrm{cm}^{2}$ & $\mathbf{R}_{\mathrm{W}} \mathbf{\Omega} . \mathrm{cm}^{2}$ & $\mathbf{T}_{\mathrm{W}} \mathbf{S e c}$ \\
& & & & \\
\hline $0.12 \mathrm{~A} / \mathrm{cm}^{2}$ & 0.16 & 0.5675 & 0.1125 & 0.003 \\
\hline $0.2 \mathrm{~A} / \mathrm{cm}^{2}$ & 0.1125 & 0.41 & 0.445 & 0.003 \\
& & & & \\
\hline $0.32 \mathrm{~A} / \mathrm{cm}^{2}$ & 0.09 & 0.3125 & 1.02 & 0.003 \\
& & & & \\
\hline
\end{tabular}

Tab. 1 Parameters of the CCL estimated through Eq. 1 


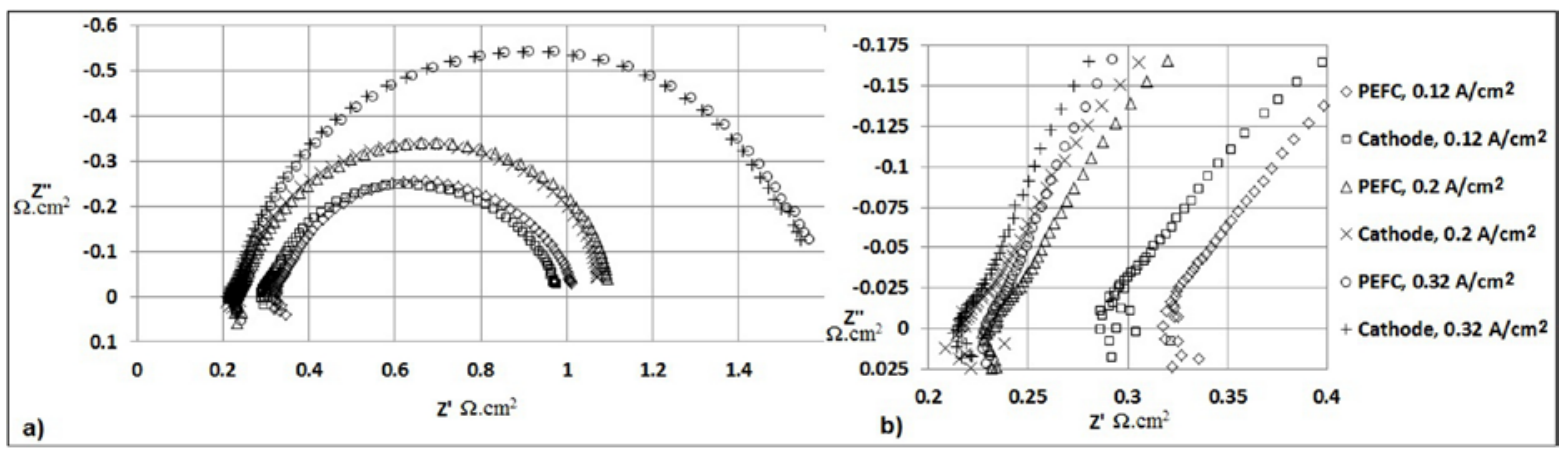

Fig. 1 a) PEFC and Cathode measured data at $0.12,0.2$ and $0.32 \mathrm{~A} / \mathrm{cm}^{2}$, b) high frequency region

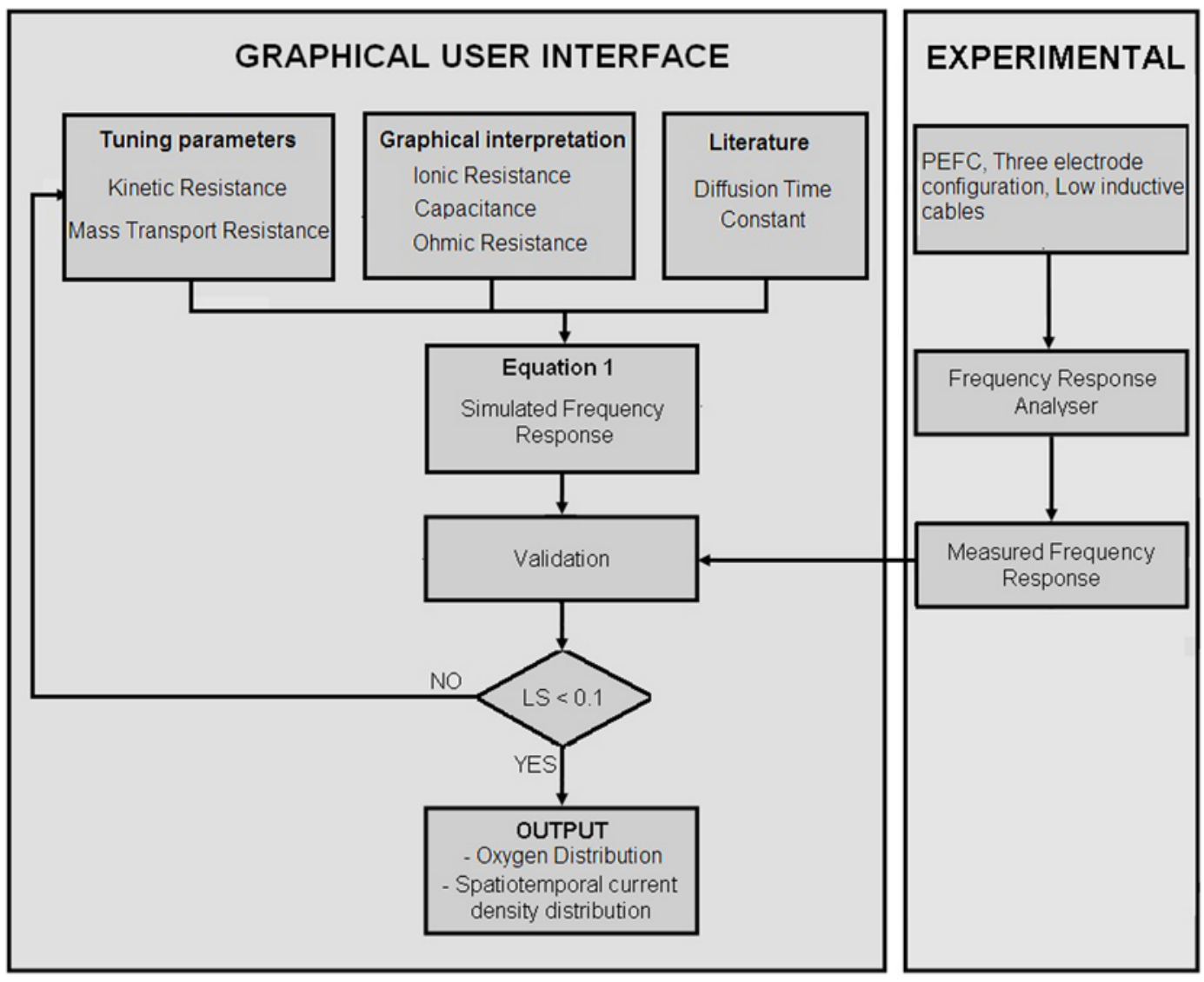

Fig. 2 Overall modelling and experimental structure 


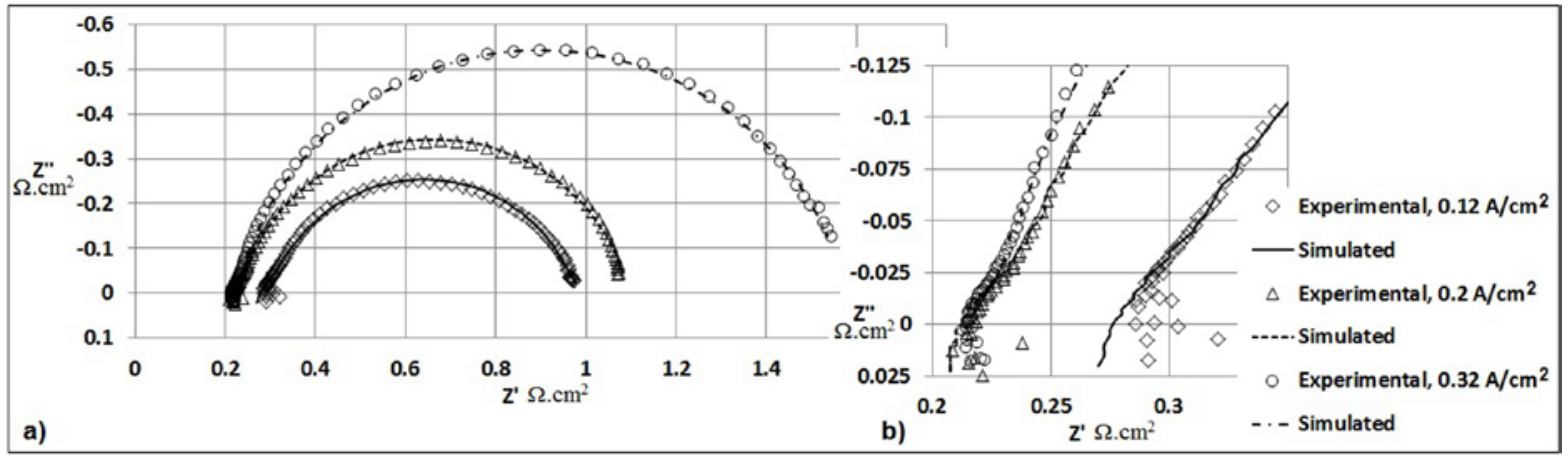

Fig. 3 a) Comparison between measured and simulated data, b) high frequency region
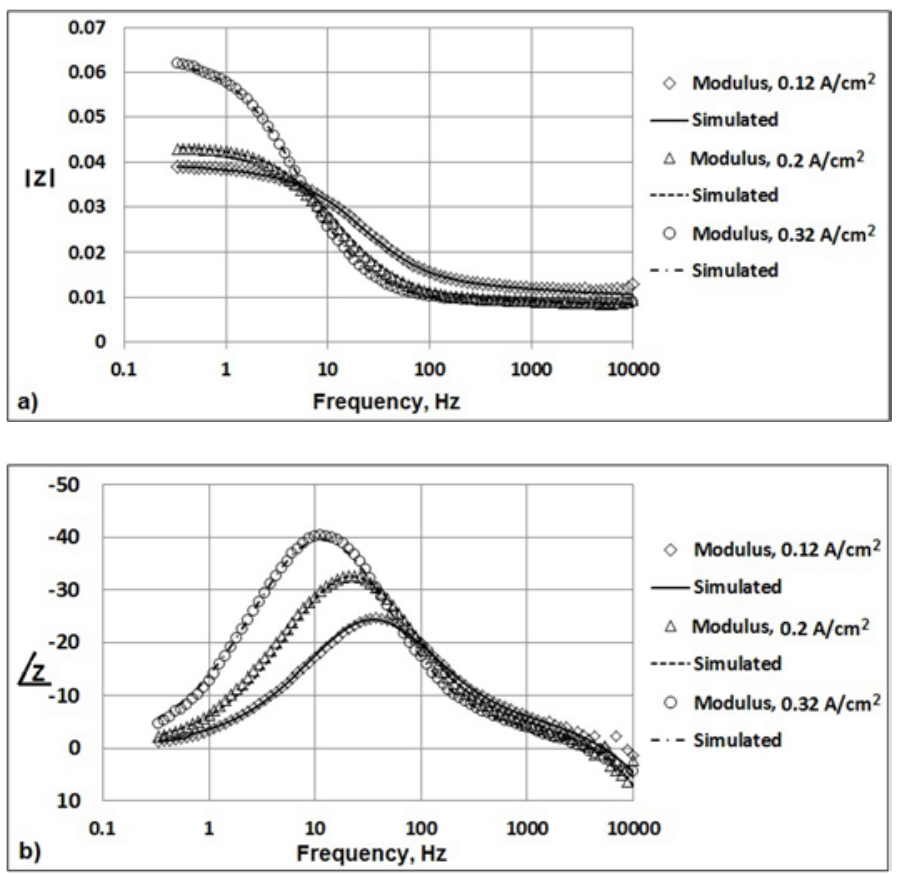

Fig. 4 Comparison in Bode format between measured and simulated data, a) modulus, b) phase angle 

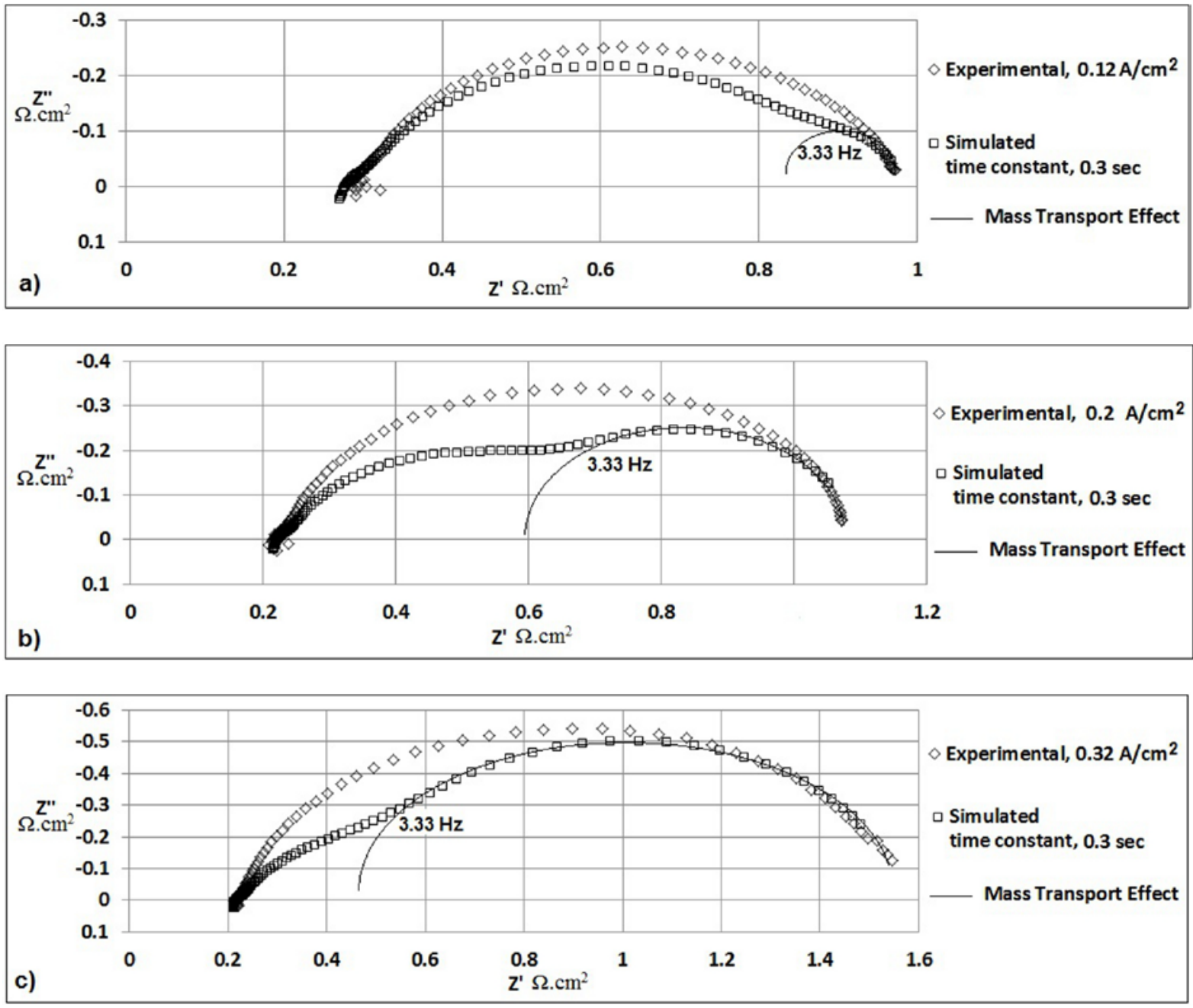

Fig. 5 Measured data with $3 m s$ time constant and simulated data with $0.3 \mathrm{~s}$ time constant, a) 0.12 $\mathrm{A} / \mathrm{cm}^{2}$, b) $0.2 \mathrm{~A} / \mathrm{cm}^{2}$, c) $0.32 \mathrm{~A} / \mathrm{cm}^{2}$
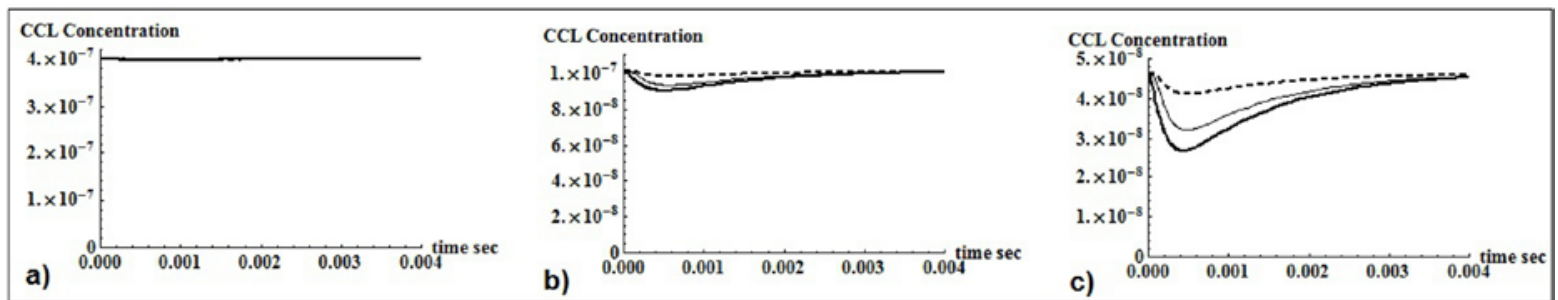

Fig. 6 Oxygen concentration distribution through the CCL thickness, dashed $x=0.25$, thin $x=0.45$, thick $x=0.75$, a) $0.12 \mathrm{~A} / \mathrm{cm}^{2}$, b) $0.2 \mathrm{~A} / \mathrm{cm}^{2}$, c) $0.32 \mathrm{~A} / \mathrm{cm}^{2}$. 


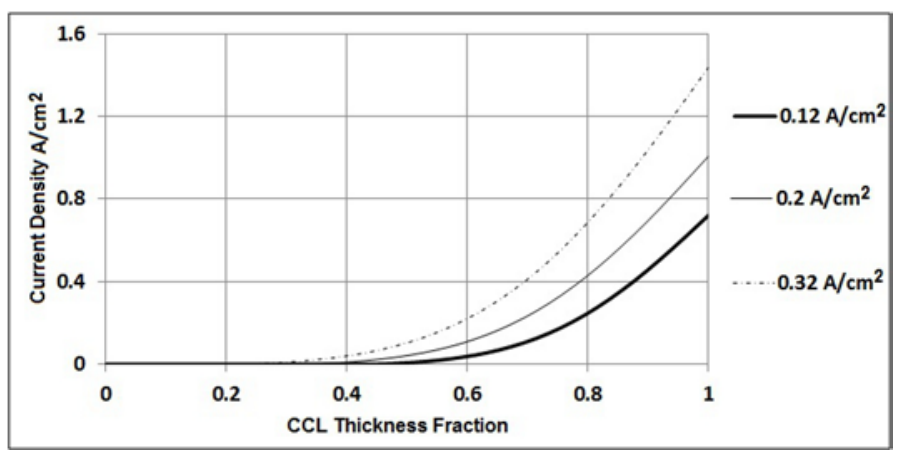

Fig. 7 Simulated current distribution through the CCL thickness

\section{References}

[1]N. Wagner, J. Appl. Electrochem. 32 (2002) 859-863.

[2]T. E. Springer, T. A. Zawodzinski, M. s. Wilson, S. Gottesfeld, J. Electrochem Soc. 143, (1996) 587-599.

[3]N. Wagner, W. Schnurnberger, B. Muller, M. Lang, Electrochim. Acta. 43, (1998) 3785-3793.

[4]T. J. P. Freire, E. R. Gonzalez, J. Electroanal. Chen. 503 (2001) 57-68.

[5]da Silva, S. L. A.; Ticianelli, E. J. Electroanal. Chem. 391 (1995) 101-109.

[6]Lee, S. J., Mukerjee, S., McBreen, J., Rho, Y. W., Kho, Y. T., Lee, T. H. Electrochim. Acta. 43, (1998) 3693-3701.

[7]V. A. Paganin, C. L. F. Oliveira, E. A. Ticianelli, T. E. Springer and E. R. Gonzalez, Electrochim. Acta. 43, (1998) 3761-3766.

[8]X. Yuan, J. C. Sun, H. Wang and J. Zhang, J. Power Sources. 161 (2006) 929-937.

[9]Perry L. Mike, Newnman J., Cirns J. E., J. Electrochem. Soc. 145 (1998) 5-15.

[10]Tsai C. R., Chen F., Ruo A.C. J. Power Sources. 160 (2006) 50-56.

[11]Y. Bultel, K. Wiezell, F. Jaouen, P. Ozil and G. Lindbergh, Electrochim. Acta. 51 (2005) 474-488.

[12]Guangyu L., Wensheng H., Trung V., J. Electrochem Soc. 151, (2004) A1999-A2006.

[13]E. L. Thompson, J. Jorne, W. Gu, H. A. Gasteiger, J. Electrochem. Soc. 155 (2008) B887-B896.

[14] S. Cruz-Manzo, P. Rama and R. Chen, J. Electrochem. Soc. 157 (2010) B1865-B1871.

[15] S. Cruz-Manzo, P. Rama and R. Chen, J. Electrochem. Soc. 157 (2010) B400-B408.

[16] R. Makharia, M. F. Mathias and D. R. Baker, J. Electrochem. Soc. 152 (2005) A970-A977 .

[17] M. Eikerling and A. A. Kornyshev, J Electroanal Chem. 475 (1999) 107-123.

[18] T. Suzuki, H. Murata, T. Hatanaka and Y. Morimoto, R\&D Review of Toyota CRDL. 39 (2004) 33-38.

[19] M. E. Orazem, N. Pebere and B. Tribollet, J. Electrochem. Soc. 153 (2006) B129-B136.

[20] N. Fouquet, C. Doulet, C. Nouillant, G. Dauphin-Tanguy and B. Ould-Bouamama, J.Power Sources., 159 (2006) 905-913.

[21] S. H. Chan, X. J. Chen, K. A. Khor, J. Appl. Electrochem. 31 (2001) 1163-1170.

[22] M. Dolle, F. Orsini, A. S. Gozdz, J. Tarascon, J. Electrochem. Soc. 148 (2001) A851-A857.

[23] Q. Yan, H. Toghiani, H. Causey, J. Power Sources. 161 (2006) 492-502.

[24] P. Gode, F. Jaouen, G. Lindbergh, A. Lundblad and G. Sundholm, Electrochim. Acta. 48 (2003) 4175-4187.

[25] F. Jaouen, G. Lindbergh and K. Wiezell, J. Electrochem. Soc. 150 (2003) A1711-A1717.

[26] S. Cruz-Manzo, P. Rama and R. Chen, ASME, J. Fuel Cell Science and Technology, under review.

[27] Ciureanu Mariana, Roberge Raymond, J. Phys. Chem. B, 105, (2001) 3531-3539.

[28] T.V. Nguyen, R.E. White, J. Electrochem. Soc. 140 (1993) 2178-2186.

[29] F. Liu, B. Yi, D. Xing, L. Yu, Z. Hou, J. Power Sources. 124 (2003) 81-89.

[30]C. H. Hsu and F. Mansfeld, Corrosion. 57 (2001) 747-748.

[31] M. Orazem, B. Tribollet, Electrochemical Impedance Spectroscopy, Wiley, New Jersey (2008).

[32]T. E. Springer, M. s. Wilson, S. Gottesfeld, J. Electrochem Soc. 140 (1993) 3513-3526.

[33]D. Malevich, E. Halliop, B. A. Peppley, J. G. Pharoah and K. Karan, J. Electrochem. Soc. 156 (2009) B216-B224.

[34] Neyerlin, K.C., Gu, W., Jorne J., Clark A., Gasteiger H., J. Electrochem. Soc. 154 (2007) B279-B287.

[35]K. Tajiri, Y. Tabuchi, C.- Y. Wang, J. Electrochem. Soc. 154 (2007) B147-B152. 